УДК 004.315

\title{
А.А. Кожевников
}

\section{Мультифункциональные арифметические устройства в остаточных классах}

\begin{abstract}
Приводится анализ некоторых подходов к формированию простейших вычислительных структур, позволяющих выполнить несколько арифметических операций в системе остаточных классов. Акцент на реализацию алгоритмов цифровой обработки сигналов после соответствующего преобразования в модулярный код инициализирует переход к функционированию устройств на основе дискретных фаз гармоник большой частоты.

Ключевые слова: система остаточных классов, арифметические устройства
\end{abstract}

doi: $10.21293 / 1818-0442-2018-21-4-59-62$

Повышение быстродействия цифровой обработки сигнала (ЦОС) возможно за счет внедрения вычислительных средств на основе системы остаточных классов [1-3] (СОК). В ряде случаев такой подход также позволяет снизить информационную избыточность в узлах спецпроцессоров [4]. Дальнейшее развитие данной идеи ведет к поиску других положительных эффектов при формировании соответствующих вычислительных структур. Базовая арифметика ЦОС в СОК строится на сложении (вычитании) и умножении, и может выполняться в параллельных трактах без обращения значений вычетов друг к другу [5]. Малость величины остатков позволяет реализовать унифицированные устройства с выигрышем в аппаратуре и быстродействии по сравнению с монофункциональными. Целью работы является анализ основных подходов к формированию структур, позволяющих выполнять несколько различных арифметических операций.

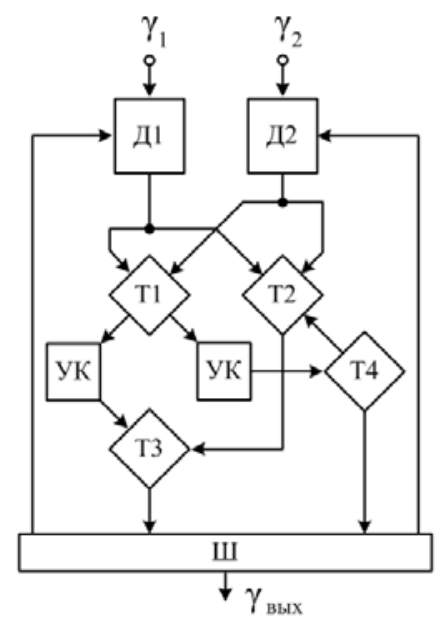

\section{Цифровые устройства}

Ряд алгоритмов, лежащих в основе логики синтеза многофункциональных устройств, использует известное выражение для вычисления произведения двух операндов, которое получается из квадратов суммы и разности:

$$
a \cdot b=\frac{1}{4}\left[(a+b)^{2}-(a-b)^{2}\right] .
$$

Рассмотрим структуру, представленную в работе [6] (рис. 1, а). Здесь Д1 и Д2 - дешифраторы; T1-Т4 - табличные вычислители; УК - умножение на константу, Ш - шифратор. Для выполнения модульного действия соотношение (1) принимает вид

$$
\left(\gamma_{a} \cdot \gamma_{b}\right) \bmod m=
$$

$$
=\left[\frac{1}{4}\left(\gamma_{a}+\gamma_{b}\right)^{2} \bmod m-\frac{1}{4}\left(\gamma_{a}-\gamma_{b}\right)^{2} \bmod m\right] \bmod m,
$$

где $m$ - модуль операции, $\gamma_{a}, \gamma_{b}$ - операнды-вычеты.

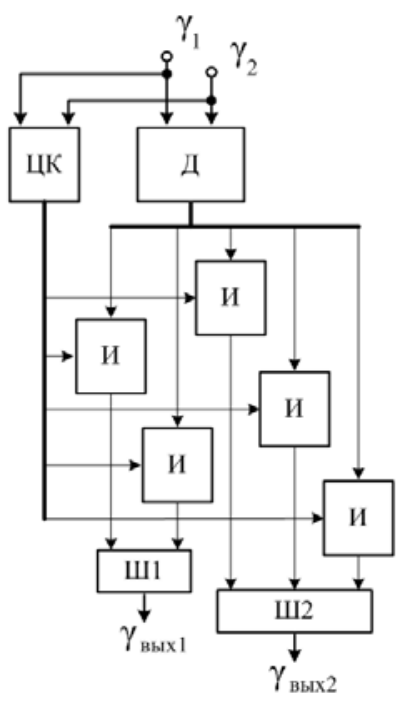

6

Рис. 1. Обобщенные схемы арифметических устройств: $a-[6], \sigma-[7,8]$

С целью уменьшения аппаратурных затрат в работе предлагается использовать внутренний модуль $k \approx \sqrt{m}$, при этом сумма и разность входящих остатков представляется в виде

$\left(\gamma_{a}+\gamma_{b}\right) \bmod m=\left[k \cdot\left(\gamma_{a}^{\prime}+\gamma_{b}^{\prime}\right)+\left(\gamma_{a}^{\prime \prime}+\gamma_{b}^{\prime \prime}\right)\right] \bmod m$,

$\left(\gamma_{a}-\gamma_{b}\right) \bmod m=\left[k \cdot\left(\gamma_{a}^{\prime}-\gamma_{b}^{\prime}\right)+\left(\gamma_{a}^{\prime \prime}-\gamma_{b}^{\prime \prime}\right)\right] \bmod m$, где $\gamma_{a}^{\prime}=\left[\gamma_{a} / k\right], \gamma_{b}^{\prime}=\left[\gamma_{b} / k\right], \gamma_{a}^{\prime \prime}=\gamma_{a} \bmod k, \gamma_{b}^{\prime \prime}=\gamma_{b} \bmod k$.

Для выполнения операции сложения (вычитания) дешифраторы Д1 и Д2 преобразуют входные величины к виду $\gamma^{\prime}$ и $\gamma^{\prime \prime}$, которые поступают на Т1 и Т2 соответственно. Далее через блоки УК, Т3 и Т4 идет выполнение (2) с восстановлением размерно- 
сти итогового вычета. Реализация операции умножения по модулю включает в себя предыдущие шаги, где дополнительно в Ш подключаются схемы квадраторов, возвращающих полученные значения обратно в Д1 и Д2, после чего осуществляется тот же алгоритм с дополнительным делением итогового значения на четыре. Последнее действие производится умножением на $Y$ - обратную мультипликативную величину числа 4 , которая определяется из уравнения $|Y \cdot 4|_{m}=1$. Например, для $m=61$ имеем $|Y \cdot 4|_{61}=1$, откуда $Y=46$.

Другой подход к реализации многофункциональных устройств основан на идее объединения в единой конструкции операций умножения и возведения в произвольную степень. Для этого авторы [7, 8] опираются на возможности бинарных манипуляций с унитарным кодом остатков в СОК. Основной фокус представленного в публикациях способа смещен на логику вычислений степенной зависимости, поскольку ее табличная реализация имеет особенности в симметрии по сравнению с умножением. Здесь (см. рис. 1, б) входные операнды попадают на дешифратор (Д) и цифровой компаратор (ЦК), по результатам работы которых активируются определенные табличные элементы на основе групп конъюнкции (И), и через шифраторы (Ш1 и Ш2) формиру-

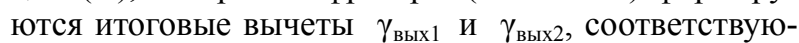
щие заданным функциям.

Тональные устройства

Известные АЦП в СОК показали свою слабость по сравнению с привычными позиционными устройствами, что выражается как через пониженное быстродействие $[9,10]$, так и точность преобразования [11]. Требуется другая парадигма, основанная на представлении вычетов в виде дискретных фаз тональных сигналов большой частоты. Соответственно и алгоритмы ЦОС должны быть перенесены на вычислительные структуры, отвечающие предложенному формату $[12,13]$. Прямой подход к решению задачи многофункциональности арифметических устройств заложен в табличном построении [14]. Здесь в качестве элементов памяти выступают управляемые фазовращатели, а доступ к конкретной ячейке осуществляется адресацией через фазированные ключи [15].

Реализация конструкции на основе как алгоритма умножения двух операндов (1), так и табличного способа вычислений определяет не только многофункциональность устройства, но и повышенное быстродействие.

Перепишем (1):

$$
4 a b=(a+b)^{2}-(a-b)^{2} .
$$

C учетом дискретно-фазированного представления по модулю $m$ (3) предстанет в виде

$$
\begin{gathered}
\frac{2 \pi}{m} \cdot\left(4 \gamma_{a} \gamma_{b}\right) \bmod m= \\
=\frac{2 \pi}{m}\left(\gamma_{a}+\gamma_{b}\right)^{2} \bmod m-\frac{2 \pi}{m}\left(\gamma_{a}-\gamma_{b}\right)^{2} \bmod m,
\end{gathered}
$$

где $\gamma_{a}$ и $\gamma_{b}$ - вычеты чисел $a$ и $b$ по модулю $m$. В правой части для суммы и разности входных операндов произведем замену так, что

$$
\left\{\begin{array}{l}
\left(\gamma_{a}+\gamma_{b}\right) \bmod m=\left(2 \gamma_{c 1}\right) \bmod m, \\
\left(\gamma_{a}-\gamma_{b}\right) \bmod m=\left(2 \gamma_{c 2}\right) \bmod m,
\end{array}\right.
$$

тогда (4) примет вид

$$
\begin{gathered}
\frac{2 \pi}{m} \cdot\left(\gamma_{a} \gamma_{b}\right) \bmod m= \\
=\frac{2 \pi}{m}\left(\gamma_{c 1}\right)^{2} \bmod m-\frac{2 \pi}{m}\left(\gamma_{c 2}\right)^{2} \bmod m .
\end{gathered}
$$

Если использовать результаты сложения и вычитания исходных операндов в качестве адресов для выбора набегов по фазе из заданных для функции умножения и равных $\frac{2 \pi}{m}\left(\gamma_{c}\right)^{2} \bmod m, \gamma_{c} \in[0 ; m-1]$, то останется только последнее вычитание фаз для окончательного выполнения алгоритма.

Рассмотрим пример. На соответствующие входы устройства подаются гармоники одной частоты (рис. 2):

$$
\begin{aligned}
& \text { - синхронизирующий } S_{0}=\sin (\omega t), \\
& \text { - первый операнд } S_{1}=\sin \left(\omega t+2 \pi \cdot \gamma_{a} / m\right) \text {, } \\
& \text { - второй операнд } S_{2}=\sin \left(\omega t+2 \pi \cdot \gamma_{b} / m\right) \text {, }
\end{aligned}
$$

где $\gamma_{a}$ и $\gamma_{b}-$ вычеты по модулю $m$, над которыми осуществляется необходимая операция. В качестве иллюстрации работы устройства рассмотрим три функции: вычитание, умножение и сумму квадратов двух операторов по модулю $m$.

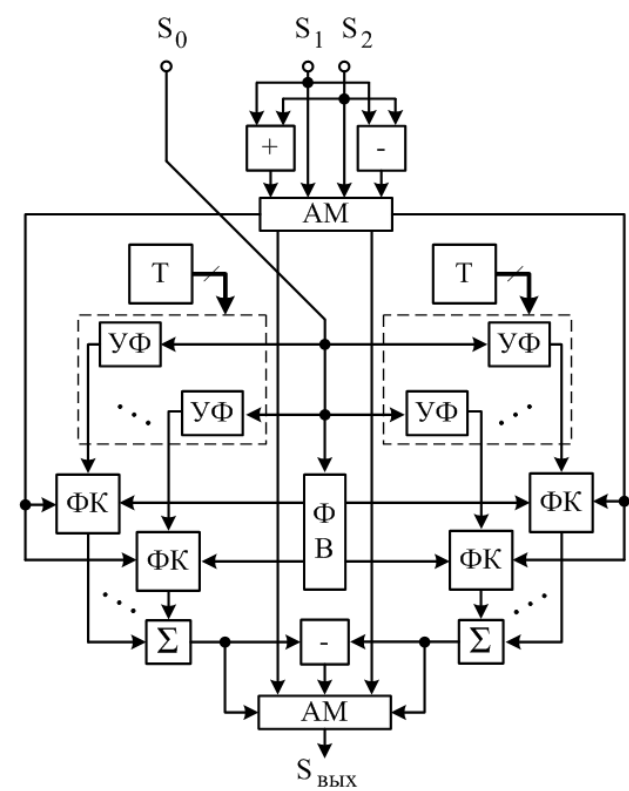

Рис. 2. Тональное арифметическое устройство

\section{1. Вычитание.}

Выход первого блока разности фаз «-» через аналоговые мультиплексоры (АМ) коммутируется непосредственно к выходу. Простейшие операции с дискретными фазами рассмотрены в [12]. После работы соответствующего блока разности на выходе наблюдается сигнал

$$
S_{\text {вых }}=\sin \left[\omega t+2 \pi \cdot\left(\gamma_{a}-\gamma_{b}\right) / m\right] \text {. }
$$


2. Умножение

Сумматор фаз «+» и первый блок разности фаз «-» через первый АМ коммутируются на входы фазированных ключей (ФК) соответственно первой и второй группы. Управляемые фазовращатели (УФ) настраиваются в соответствии с таблицами в ПЗУ (Т) для операции умножения на набеги фазы $\frac{2 \pi}{m}\left(\gamma_{c}\right)^{2} \bmod m$, где $\gamma_{c} \in[0 ; m-1]$. Второй АМ подключает выход устройства к второму блоку разности фаз.

Блоки «+» и «-» формируют сумму и разности фаз гармоник входных операндов:

$$
\begin{aligned}
& S_{+}=\sin \left[\omega t+2 \pi \cdot\left(\gamma_{a}+\gamma_{b}\right) / m\right], \\
& S_{-}=\sin \left[\omega t+2 \pi \cdot\left(\gamma_{a}-\gamma_{b}\right) / m\right],
\end{aligned}
$$

которые используются в качестве адреса, позволяющих пропустить сигналы от соответствующих УФ. На втором блоке разности фаз происходит окончательная реализация алгоритма, соответствующего выражению (4):

$$
S_{\text {вых }}=\sin \left[\omega t+\frac{2 \pi}{m} \cdot\left(\gamma_{a} \cdot \gamma_{b}\right) \bmod m\right] \text {. }
$$

Если рассмотреть данные вычисления на цифpax для $\gamma_{a}=2, \gamma_{b}=3$ и $m=5$, то получим следующие соотношения:

$$
\begin{gathered}
S_{+}=\sin [\omega t+2 \pi \cdot(5) / 5]=\sin (\omega t), \\
S_{-}=\sin [\omega t+2 \pi \cdot(-1) / 5]=\sin [\omega t+2 \pi \cdot 4 / 5] .
\end{gathered}
$$

Эти сигналы задают адреса для УФ, настроенных на $0 \cdot 2 \pi / 5=0$ и $4 \cdot 2 \pi / 5=8 \pi / 5$. На выходе формируется:

$$
\begin{gathered}
S_{\text {вых }}=\sin \left[\omega t+0-4 \cdot \frac{2 \pi}{5}\right]=\sin \left[\omega t+1 \cdot \frac{2 \pi}{5}\right]= \\
=\sin \left[\omega t+\frac{2 \pi}{5} \cdot(2 \cdot 3) \bmod 5\right] .
\end{gathered}
$$

\section{3. Сумма квадратов}

Входы операторов $S_{1}$ и $S_{2}$ через первый АМ коммутируются на ФК соответственно первой и второй группы. Управляемые фазовращатели настраиваются в соответствии с функцией на квадрат и минус квадрат операнда по модулю. Второй АМ подключает выход устройства ко второму блоку разности фаз.

Рассмотрим работу устройства для $\gamma_{a}=2, \gamma_{b}=3$ и $m=5$. Сигналы входных операндов используются в качестве адреса и позволяют пропустить гармоники от управляемых фазовращателей с фазами $4 \cdot 2 \pi / 5$ и $1 \cdot 2 \pi / 5$ соответственно. На втором блоке разности фаз происходит вычитание минус квадрата второго операнда из квадрата первого, формируя конечный результат:

$$
\begin{gathered}
S_{\text {вых }}=\sin \left[\omega t+\frac{2 \pi}{5} \cdot 4-\frac{2 \pi}{5} \cdot 1\right]= \\
=\sin \left[\omega t+\frac{2 \pi}{5} \cdot 3\right]=\sin \left[\omega t+\frac{2 \pi}{5} \cdot\left(2^{2}+3^{2}\right) \bmod 5\right] .
\end{gathered}
$$

Заключение

Как видно из представленных примеров многофункциональных устройств, наиболее удобными подходами здесь являются табличный и гибридный, последний из которых включает в себя предыдущий вариант, логично встроенный в конструкцию на основе алгоритма (1). Эти две основные парадигмы обоснованно занимают свое место как в цифровом формате кодов СОК, так и дискретно-фазированном. Представленные способы опираются на простоту табличной реализации и нюансы симметрии в расположении итоговых вычетов относительно входных операндов. Построение структур на основе выражения (1) позволяет повысить эффективность мультифункциональных устройств.

\section{Лuтература}

1. Ирхин В.П. Табличная реализация цифровых фильтров в модулярной арифметике / В.П. Ирхин, Л.А. Овчаренко // Информационные технологии. - 2005. №10. - C. 13-20.

2. Галанина Н.А. Анализ эффективности синтеза устройств вычислительной техники для непозиционной цифровой обработки сигналов / Н.А. Галанина, Н.Н. Иванова // Кибернетика и программирование. - 2015. - № 3. C. 1-6.

3. Овчаренко Л.А. Реализация цифрового трансверсального фильтра в системе остаточных классов / Л.А. Овчаренко // Радиоэлектроника. - 2002. - №4. C. $50-57$.

4. Мельник В.А. Информационная избыточность в узлах непозиционного спецвычислителя для телекоммуникационных устройств / В.А. Мельник, Р.В. Кузьменко, В.П. Ирхин // Вестник Воронеж. ин-та МВД России. 2015. - №2. - С. 149-155.

5. Акушский И.Я. Машинная арифметика в остаточных классах / И.Я. Акушский, Д.И. Юдицкий. - М.: Сов. радио, 1968. - $440 \mathrm{c.}$

6. Пат. 2157560 РФ, МПК G06F7/72, G06F7/49. Арифметическое устройство по модулю / В.П. Ирхин и др. - Опубл. 10.10.2000.

7. Ирхин В.П. Расширение функциональных возможностей вычислителей в телекоммуникационных устройствах / В.П. Ирхин, В.А. Мельник, Д.С. Шведов // Вестник Воронеж. ин-та ФСИН России. - 2016. - №1. - С. 21-26.

8. Пат. 2624587 РФ, МПК G06F7/49. Устройство для умножения чисел по модулю / В.П. Ирхин и др. - Опубл. 04.07.2017.

9. Кожевников А.А. Методы непозиционного аналого-цифрового преобразования / А.А. Кожевников, К.П. Беспалов // Автометрия. - 2015. - Т. 51, № 6. C. $125-130$.

10. Кожевников А.А. Синтез аналого-цифровых, первичных и вторичных модулярных измерительных преобразователей // Наука. Инновации. Технологии. - 2017. № 1. - C. 17-28.

11. Кожевников А.А. Математическое обследование конвейерных АЦП в системе остаточных классов // Вестник БГТУ. - 2017. - № 7. - С. 27-34.

12. Кожевников А.А. Арифметические вентили модулярных спецпроцессоров // Приборы и системы. Управление, контроль, диагностика. - 2018. - №2. - С. 46-51.

13. Методы построения устройств аналоговых и полуаналоговых вычислителей / А.А. Кожевников, Д.С. Шведов // Сб. матер. междунар. науч.-практ. межведом. конф. «Техника и безопасность объектов уголовноисполнительной системы». - Воронеж, 2016. - С. 258-260.

14. Пат. 2656992 РФ, МПК G06F 7/72. Арифметическое устройство по модулю $\mathrm{m} /$ А.А. Кожевников и др. Опубл. 07.05.2018. 
15. Пат. 2659866 РФ, МПК G01R 25/00, G01R 29/02, Н03К 17/00. Фазированный ключ по модулю m / А.А. Кожевников и др. - Опубл. 04.07.2018.

\section{Кожевников Алексей Александрович}

Канд. физ.-мат. наук, доцент каф. социально-гуманитарных, естественно-научных и общепрофессиональных дисциплин Ростовского государственного университета путей сообщения (РГУПС), г. Воронеж Урицкого ул., д. 75а, г. Воронеж, РФ, 394026

Тел.: +7-909-211-06-74

Эл. почта: akozhev@yandex.ru

Kozhevnikov A.A.

\section{Multifunctional arithmetic devices in residual classes}

The paper presents an analysis of some approaches to the formation of simple computational structures that allow performing several arithmetic operations in the residue number system. The emphasis on the implementation of digital signal processing algorithms after the corresponding conversion to modular code, initializes the transition to the operation of devices based on discrete phases of high frequency harmonics. Keywords: residue number system, arithmetic units. doi: $10.21293 / 1818-0442-2018-21-4-59-62$

\section{References}

1. Irkhin V.P., Ovcharenko L.A. Table representation of digital pilfers in modular arithmetic. Information Technology, 2005, no.10, pp. 13-20 (in Russ.).

2. Galanina N.A., Ivanova N.N. Analysis of the effectiveness of synthesis of computing devices for non-positional digital signal processing. Cybernetics and programming, 2015, no. 3, pp. 1-6 (in Russ.).

3. Ovcharenko L.A. Implementation of a digital transversal filter in the system of residual classes. Radio electron$i c s, 2002$, no. 4, pp. 50-57 (in Russ.).

4. Melnik V.A., Kuzmenko R.V., Irkhin V.P. Information redundancy in the nodes of the nonpositional specialty calculator for telecommunication devices. The bulletin of Voronezh Institute of the Ministry of Internal Affairs of Russia, 2015, no. 2, pp. 149-155 (in Russ.).
5. Akushskii I.Ya., Yuditskii D.I. Mashinnaya arifmetika $v$ ostatochnih klassah [Arithmetic of computers in the residue number system]. Moscow, Sov. Radio, 1968, 440 p.

6. Patent RF no. 2157560. Arithmetic device by module / Irkhin V.P. et al., Published October 10, .2000 (in Russ.).

7. Irkhin V. P., Melnik V.A., Shvedov D.S. The expansion functionality of calculators in telecommunication devices. Proceedings of Voronezh institute of the Russian federal penitentionary service, 2016, no.1, pp. 21-26 (in Russ.).

8. Patent RF no.2624587. Device for multiplying by module / Irkhin V.P. et al. Published July 4, 2017. (In Russ.).

9. Kozhevnikov A.A., Bespalov K.P. Nonpositional analog-digital convertion methods. Avtometriya, 2015, no. 6, pp. 125-130 (in Russ.).

10. Kozhevnikov A.A. The synthesis of analog-todigital, primary and secondary modular converters. Science. Innovations. Technologies, 2017, no. 1, pp. 17-28. (in Russ.).

11. Kozhevnikov A.A. Mathematical investigation of conveyer analog-digital convertions in the system of residual classes. Bulletin of Bryansk State Technical University, 2017, no. 7, pp. 27-34/

12. Kozhevnikov A.A. Arithmetic simple devices of modular special processors. Instruments and Systems: Monitoring, Control, and Diagnostics, 2018, no. 2, pp. 46-51 (in Russ.).

13. Kozhevnikov A.A., Shvedov D.S. Metody postroeniya ustrojstv analogovyh i poluanalogovyh vychisliteley [Methods for building devices of analog and semi-analog computers]. Sbornik materialov Mezhdunarodnoy nauchnoprakticheskoy mezhvedomstvennoy konferentsii Tekhnika i bezopasnost' ob"ektov ugolovno-ispolnitel'noj sistemy [Materials of the International Scientific and Practical Interdepartmental Conference Technique and safety of objects of the penitentiary system]. Voronezh, 2016, pp. 258-260.

14. Patent RF no.2656992. Arithmetic device by m module / Kozhevnikov A.A. et al. Published May 7, 2018 (in Russ.).

15. Patent RF no.2659866. Phased key modulo m / Kozhevnikov A.A. et al. Published July 4, 2018 (in Russ.).

\footnotetext{
Alexey A. Kozhevnikov

Associate professor of the

Department Social, Human, Natural Sciences and General Professional Disciplines,

Rostov State Transport University, Voronezh city

75a, Uritskogo st., Voronezh, Russia, 394026

Phone: +7-909-211-06-74

Email: akozhev@yandex.ru
} 